\title{
An adapted ODE model to study the Dynamics of SARS-Cov-2 Infection (COVID-19): different scenarios for Brazil and other countries
}

Ramón E. R. González ( $\square$ ramon.ramayo@ufrpe.br)

Rural Federal University of Pernambuco

Miguel A. Zorro M.

Rural Federal University of Pernambuco

C. Estupiñán-López

Federal University of Pernambuco

Carlos Andrés Collazos Morales

University Manuela Beltrán

Research Article

Keywords: SARS-Cov-2, transmissibility model, scenario comparisons

Posted Date: May 20th, 2020

DOI: https://doi.org/10.21203/rs.3.rs-29563/v1

License: (c) (i) This work is licensed under a Creative Commons Attribution 4.0 International License.

Read Full License 


\title{
An adapted ODE model to study the Dynamics of SARS-Cov-2 Infection (COVID-19): different scenarios for Brazil and other countries.
}

\author{
Ramón E. R. González ${ }^{1, *}$, Miguel A. Zorro M. ${ }^{2,+}$, C. Estupiñán-López ${ }^{3,+}$, and Carlos \\ Andrés Collazos Morales ${ }^{4,+}$
}

\author{
${ }^{1}$ Laboratory of Complex Systems and Universalities, Department of Physics, Rural Federal University of \\ Pernambuco, 52171-900, Recife, Pernambuco, Brazil. \\ ${ }^{2}$ Academic Unit of Cabo de Santo Agostino, Rural Federal University of Pernambuco, 54519-430, Cabo de Santo \\ Agostino, Pernambuco, Brazil. \\ ${ }^{3}$ Laboratory of Biomedical Optics and Imaging, Federal University of Pernambuco, 50670-901, Recife, PE, Brazil. \\ ${ }^{4}$ Research Group in Basic Sciences and Laboratories, University Manuela Beltrán, 111166, Bogotá, Colombia. \\ *ramon.ramayo@ufrpe.br \\ +these authors contributed equally to this work
}

\begin{abstract}
A mathematical model to calculate the transmissibility of SARS-Cov-2 in Wuhan City was developed and published recently by Tian-Mu Chen et al. The present work generalizes this model to study the effect of different scenarios that include actions to contain the pandemic, such as the isolation and quarantine of infected and at-risk people. Comparisons made between the different scenarios show that the progress of the infection depends heavily on the measures taken in each case. The particular case of some countries, was studied, showing the dynamics of infection in comparison with the different scenarios contained in the model. Real data from different countries, such as Brazil, was used for the validation of the model. This study shows that the model reproduce adequately the dynamic of infection for all studied countries. The relative evolution of the number of new infections and reported cases was used to estimate a pandemic containment date. The progression of the epidemic was analyzed using the system's phase diagram, which shows the "path" followed by the epidemic in each scenario. Finally, the basic values of the number of reproduction $R_{0}$ were estimated for each scenario, ranging from 4.04 to 1.12.
\end{abstract}

\section{Introduction}

On February 26, the first case of SARS-Cov-2 was reported in Brazil ${ }^{1}$. From that day on, and taking into account the spread of the epidemic in China and Europe, a group of measures began to be taken, aiming to minimize the effects of what was later classified by the World Health Organisation (WHO) as a global pandemic. The scientific community, playing its role in the midst of this global emergency, has focused its efforts on studying, among other things, the dynamics of the spread of the virus in several countries. For many years ago, epidemics have been modeled using differential equations, cellular automata and complex networks ${ }^{2-14}$. Dengue, influenza, malaria, tuberculosis, among others, are some of the epidemics that have been studied using these models. Several studies disseminating models that study the dynamics of the epidemic have been carried out and published in these months from the first reported case ${ }^{15-19}$. One of the first was published by Tian-Mu Chen et al., Infectious Diseases of Poverty, 2020 ${ }^{15}$. In that work, the Bats-Hosts-Reservoir-People transmission network model was developed to simulate the potential transmission of the source of infection (likely bats) to humans. We developed a new version of the model that only a person-to-person transmission is considered, as this is the dynamic for the rest of the countries, except China, where the epidemic emerged. The new version is a simple model of the SIR type, which includes some characteristic variables of the containment measures that have been taken worldwide. Several parameters used in the model simulate biological characteristics of the virus. Others are control parameters in the adjustment of the model to the reality of the countries studied. Despite the fact that the dynamics of the epidemic show similar qualitative behavior in any country, the specific characteristics of each region play a relevant role in the simulation process. The basic reproduction rate $R_{0}$ is defined from the parameters used in the model. The model is applied to different scenarios, defined by different degrees of adherence to containment measures of the epidemic. To demonstrate the importance of these measures, comparisons of the dynamics of virus infection and spread are made. The studies carried out are based on the morphological characteristics of the infection curves, such as the height of the infection peak, its position and its width. Within each simulated scenario, the basic reproduction rate is 
calculated. $R_{0}$ values are used to compare the different scenarios in order to define which one is the most promising. Another important aspect of the study being carried out is the analysis of the progress of the epidemic from a phase diagram and the comparison between possible behaviors for the different countries involved in the study based on the model presented in this work.

\section{Results}

Temporal evolution of the epidemic: four different scenarios.

From the results of the simulations made using our model, we were able to compare the peaks of infection in different scenarios. Figure 1. shows the total number of the infected individuals as a function of time for the 4 different scenarios. These curves are solution of the systems of equations described in Methods section. This scenarios can be related to the real spread of the disease in countries around the globe. Taking Brazil as our main subject of study. The most pessimistic scenario (scenario 0) corresponds to that in which no containment measure is taken. In this scenario, the number of infected in Brazil can reach more than a million, and the peak of infection will appear in a short time, in may 04, approximately 69 days after February 24, when the first case in the country was reported. The delay of the peak beyond what was foreseen for scenario 0 would be an important and decisive factor for the health system of each country to satisfy the demand of patients with COVID-19. On the other hand, scenario 3, which is the most promising, is characterized by a much less significant peak. The peak is dislocated to the right, which means that in this scenario there would be enough time to prepare the health system for the efficient confrontation of the pandemic. In Figure 1. the real data of Brazil is also plotted as a method to compare with the 4 scenarios found with our model and in Table 1. a summary of the characteristic values of each scenario is shown.

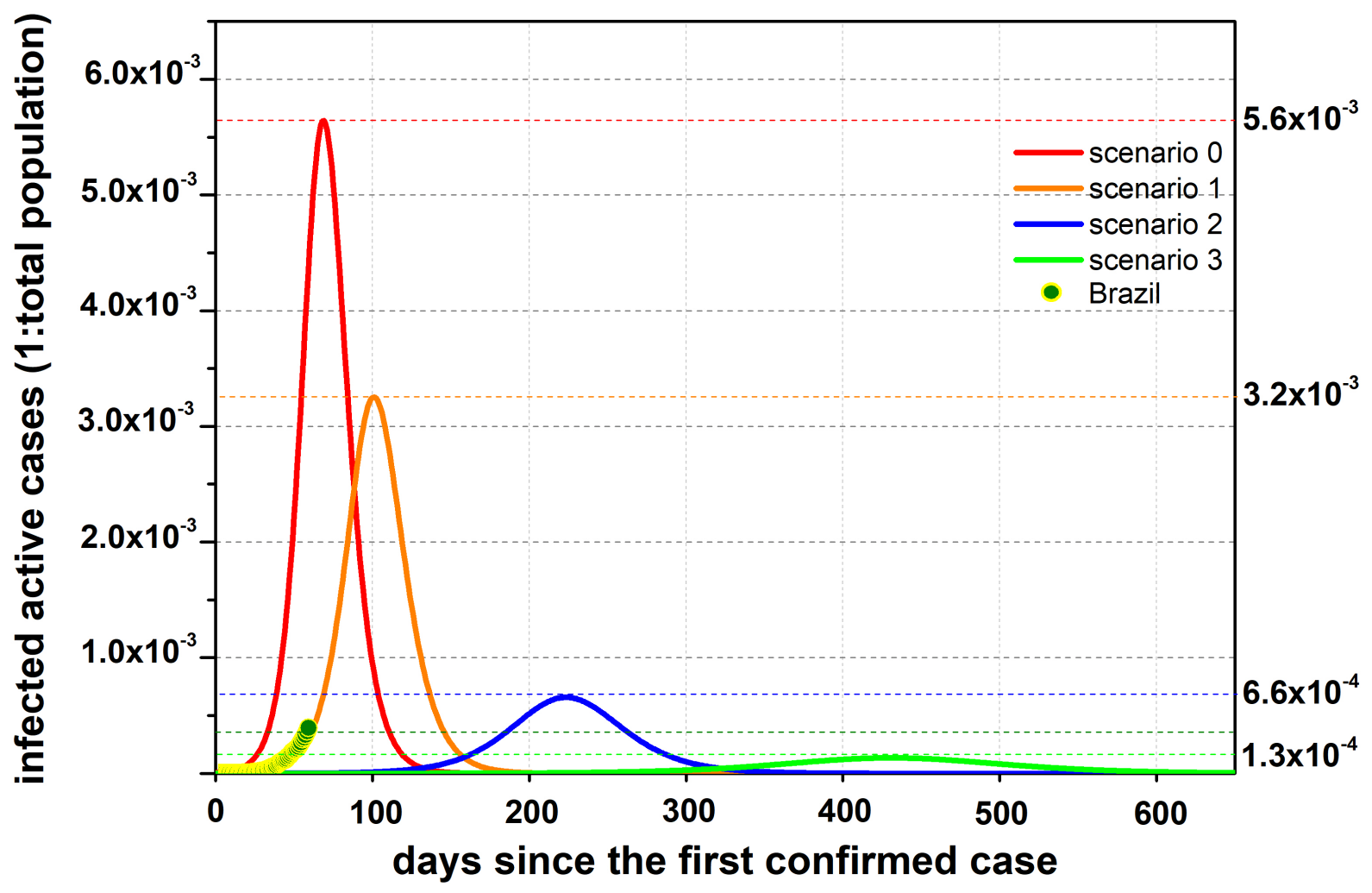

Figure 1. Total infected cases versus time in days, for all scenarios. In red, the dynamic corresponding to the worst scenario is represented, while the most optimistic scenario is represented in green. Vertical axis represent the reported values of infected cases. Real data for Brazil until April 30 are represented with coloured circles. The control parameters used in simulations was: $\beta=0.8, \phi=10^{-4}$, and for the isolation rate was used $\sigma=0.1$. 


\begin{tabular}{|l|l|l|c|}
\hline Scenario & Peak height & Peak position & Duration \\
\hline 0 & 564045 & 69 days & 106 days \\
\hline 1 & 256982 & 100 days & 112 days \\
\hline 2 & 51301 & 222 days & 139 days \\
\hline 3 & 13475 & 431 days & 242 days \\
\hline
\end{tabular}

Table 1. Characteristic values of peaks of infection in different scenarios.

\section{Exponential growth.}

Actual data ${ }^{20}$ on SARS-Cov-2 infected cases have been reported by the Ministry of Health and the state departments since the beginning of the epidemic. The graph in Figure 2 shows, in the left panel, the dynamics of the initial infection curve for each simulated scenarios and the curve of real cases in some countries, for the first weeks of epidemic, and in the right panel, the curve for Brazil. Here it is interesting the fact that we cannot adapt any country to a single scenario, however, we can say that the epidemic behaves in all the countries studied, among the four simulated scenarios considered in the model. Corona-virus infection is a dynamic process that undergoes changes due to the containment measures that are being adopted. This means that the behavior of the real data is a composition of several separate scenarios, with the particularities of each country. Analyzing the graph we can see how there are different growth regimes for each country. In general, the initial stages begin with a growth in the order of scenarios 0 or 1 approximately, this possibly due to the fact that the containment measures have not yet been strengthened. Already in the later stages, after the measures adopted, in general terms, slower growth of the infected cases are observed, and it's possible to remark that the real data of the countries behaves going through a growth rate similar to the scenario 2 and later to the scenario 3 (see Figure 2, right panel). After the exponential grow, China, South Korea, and Norway show stability, which represents a drop in the new cases reported in these three countries, as well as Spain and Italy but this ones in the early days of the stability stage, as can be seen in Figure 2. Table 2 below lists the growth rates for the four scenarios and the actual reported data. To better understand the comparison of each country with the different scenarios, the panels in the Figure 3. represent the six countries under study that reached or passed the peak of infection. In most of the countries studied, the beginning of the epidemic had a rather atypical behavior in relation to the reported infected cases. From the analysis of these graphs, we see that by the time stability is reached, all the countries are close to scenario 2 . This means that this is the scenario to be reached by the countries that have not yet reached their peak of infection. On the other hand, the transition from an initial and unfavorable scenario to a more promising one occurs in different ways and at different times in each country. USA takes approximately 65 days to transit from scenario 1 to 2 , while Norway take much less time, 13 days approximately.

\begin{tabular}{|l|l|}
\hline Scenario/Country & Grow rate \\
\hline 0 & 0.15 \\
\hline 1 & 0.13 \\
\hline 2 & 0.06 \\
\hline 3 & 0.03 \\
\hline Brazil & $\sim 0.13$ \\
\hline China & $\sim 0.16$ \\
\hline South Korea & $\sim 0.15$ \\
\hline Italy & $\sim 0.12$ \\
\hline Spain & $\sim 0.14$ \\
\hline Norway & $\sim 0.16$ \\
\hline USA & $\sim 0.12$ \\
\hline
\end{tabular}

Table 2. Growth rates of exponential curves for different scenarios compared to the actual date of cases reported in some countries for the first weeks of the pandemic.

\section{New cases versus active cases.}

Recently ${ }^{21}$, interesting results have been reported showing methods that make it possible to clearly distinguish the end of exponential growth, depending on the total number of reported cases compared to daily new cases. The graph in Figure 4 shows a study of this behaviour, based on real data ${ }^{20}$, and in the simulation results. In this graph, we can see the growth of new cases in relation to the total reported cases. This growth is approximately the same in all countries. China, South Korea, Norway, Spain, and Italy are the countries that have been showing a drastic decline in the number of new cases reported. This implies that these countries have already exceeded the peak of infection. We can also see that the United States is apparently 


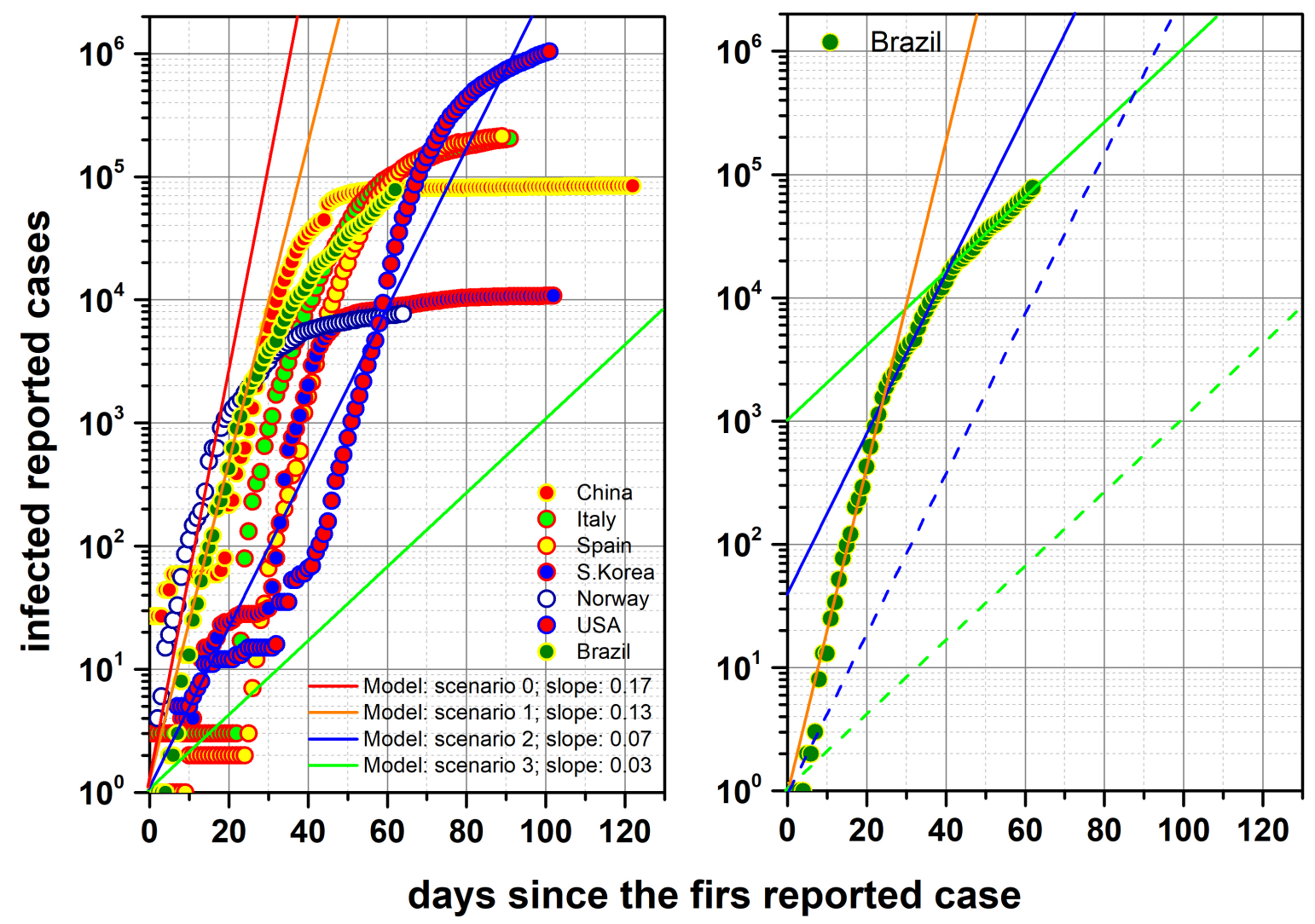

Figure 2. Representative curves of the exponential growth. The colours from red to green represent scenarios $0,1,2$ and 3 respectively. Real confirmed cases for each country ${ }^{20}$ are represented with different coloured circles, in the left panel, and for Brazil in the right panel. The control parameters used in simulations was: $\beta=0.8, \phi=10^{-4}$, and for the isolation rate was used $\sigma=0.1$.

very close to that moment. As seen at the right panel of Figure 4., our model behaves the same as real data, with a growth rate of approximately 0.98 , the same in all scenarios, while the real growth of new cases occurs at a rate of 0.90 . From the comparison between the two graphs, USA is in the worst case scenario among all the countries, at the moment that the cases reported daily start to fall, while Norway and South Korea show the best scenarios. When trying to locate the countries studied in a certain scenario, from the comparison between the two graphs in the Figure 4., we noticed a small difference in relation to what had been concluded from the analysis of the exponential growth of the epidemic for the different countries. Different criteria are used for these classifications. Growth rates calculated above represent the temporal dynamics of the epidemic at different stages of its evolution. Near the moment when the number of new cases begins to decrease, a change in the behavior of the epidemic curve occurs, causing this difference between the two criteria.

\section{Parametric curves.}

The study of the parametric curves from behavior of the number of susceptible elements over time as a function of the active infected elements provides a different and very interesting vision of the spread of the epidemic. The end of the epidemic occurs when there are no more active infected cases. At that time, in any scenario there will always be a population of susceptible elements, those that the epidemic did not reach. Figure 5 represents the "phase diagram" to the spread of the disease. For each scenario, there is a "critical" value for the number of susceptible individuals. This number represents the smallest number of susceptible individuals necessary for the spread of the epidemic. This value depends on each scenario. For more pessimistic scenarios, the number of susceptible individuals at the end of the epidemic is lower, due to the greater number of people who will come into contact with the virus. The real data reported by the World Health Organization (WHO) allow us to speculate in relation to what the possible behaviors of these curves could be for each country under analysis. The infection peaks of the 


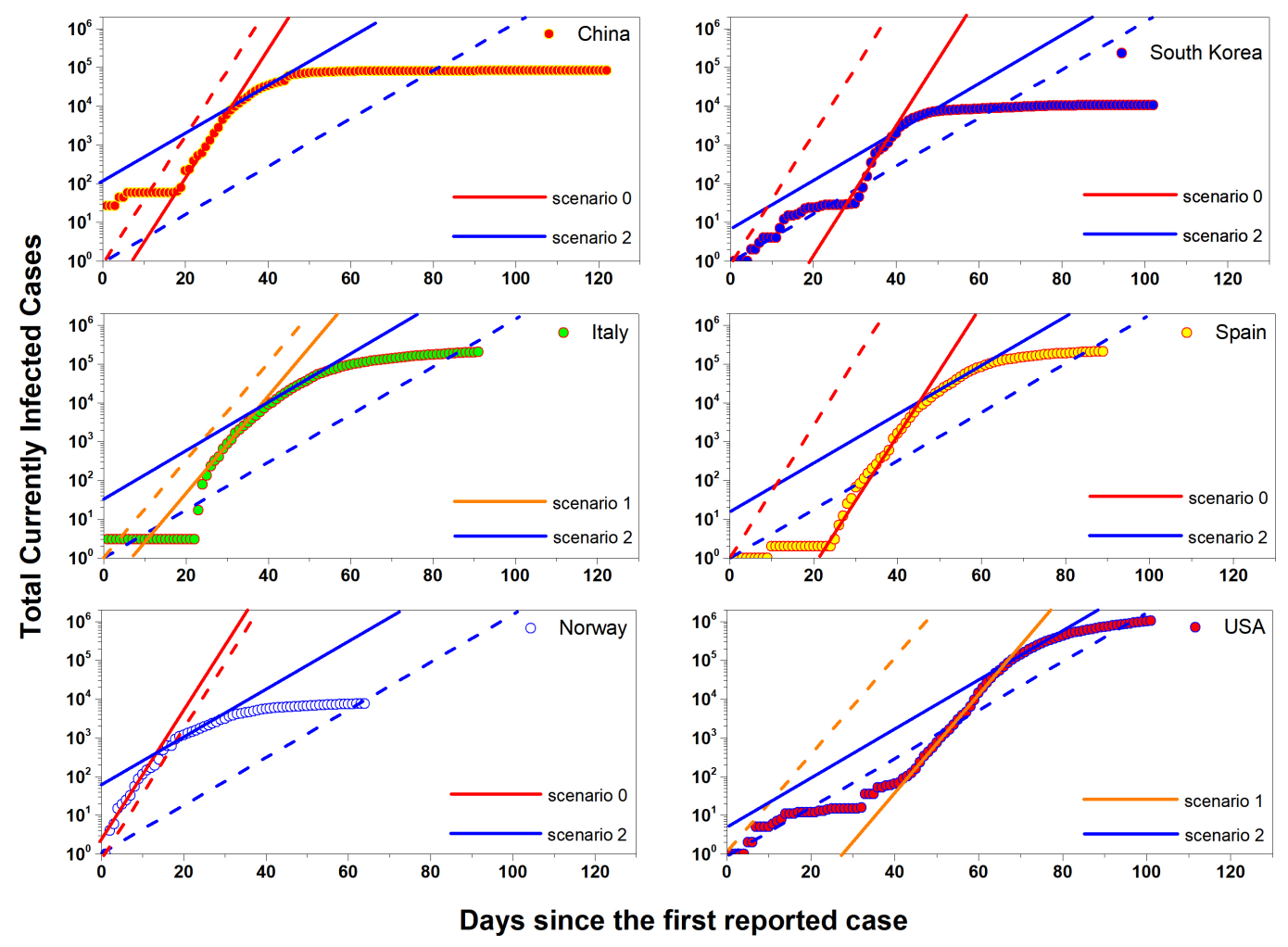

Figure 3. Representative curves of the exponential growth. Different countries are represented in each panel. The scenarios corresponding to each stage of progression of the epidemic are represented with colored lines. The control parameters used in simulations was: $\beta=0.8, \phi=10^{-4}$, and for the isolation rate was used $\sigma=0.1$.

countries under study are represented on the phase diagram by colored circles. These circles are on the imaginary line that joins the maximums corresponding to each scenario in the phase diagram. Analyzing these points, corresponding to real data, and using the results of the simulations, we can locate each country within the specific scenario, or between two of them, at the time of the peak of infection. The number of susceptible individuals depends on the population of each country. If we analyze the behavior of the epidemic in the countries studied based on the proportion of infected people in relation to the total population, the situation in the countries is different from what was seen in previous sections. China, for example, was classified between scenarios 0 and 2 based on the growth rate of the epidemic and the number of new cases at the time of the peak. This further classification places it in scenario 3. Norway, in the best scenario in relation to the new cases reported at the peak of the epidemic, is now located between scenarios 1 and 2.

\section{Basic Reproduction Rate.}

The basic reproduction number $R_{0}$ is an important parameter when it comes to studying epidemics and their dynamics of infection. From the parameters of our model, including all possible scenarios, we can define $R_{0}$ in equation (1). In this expression, $i$ represent a number of scenario. $R_{0}$ was estimated in all the scenarios and for all the values of $\sigma$. The values of the basic reproduction numbers in each simulated scenario, using $\beta=0.8$, are shown in Table 3. From the values of the basic reproduction rate obtained in the simulations, we can see that in terms of $R_{0}$ there are scenarios that can be equivalent depending on the characteristic parameters. Scenario 1 , using $\sigma=0.3$, is equivalent to scenario 3 , with $\sigma=0.1$, as well as scenario 2 with $\sigma=0.3$ is equivalent to 3 with $\sigma=0.2$. It is possible that the fact that one scenario is more promising than another, in relation to the number of measures, is conditioned on strict compliance and efficiency of these measures. 


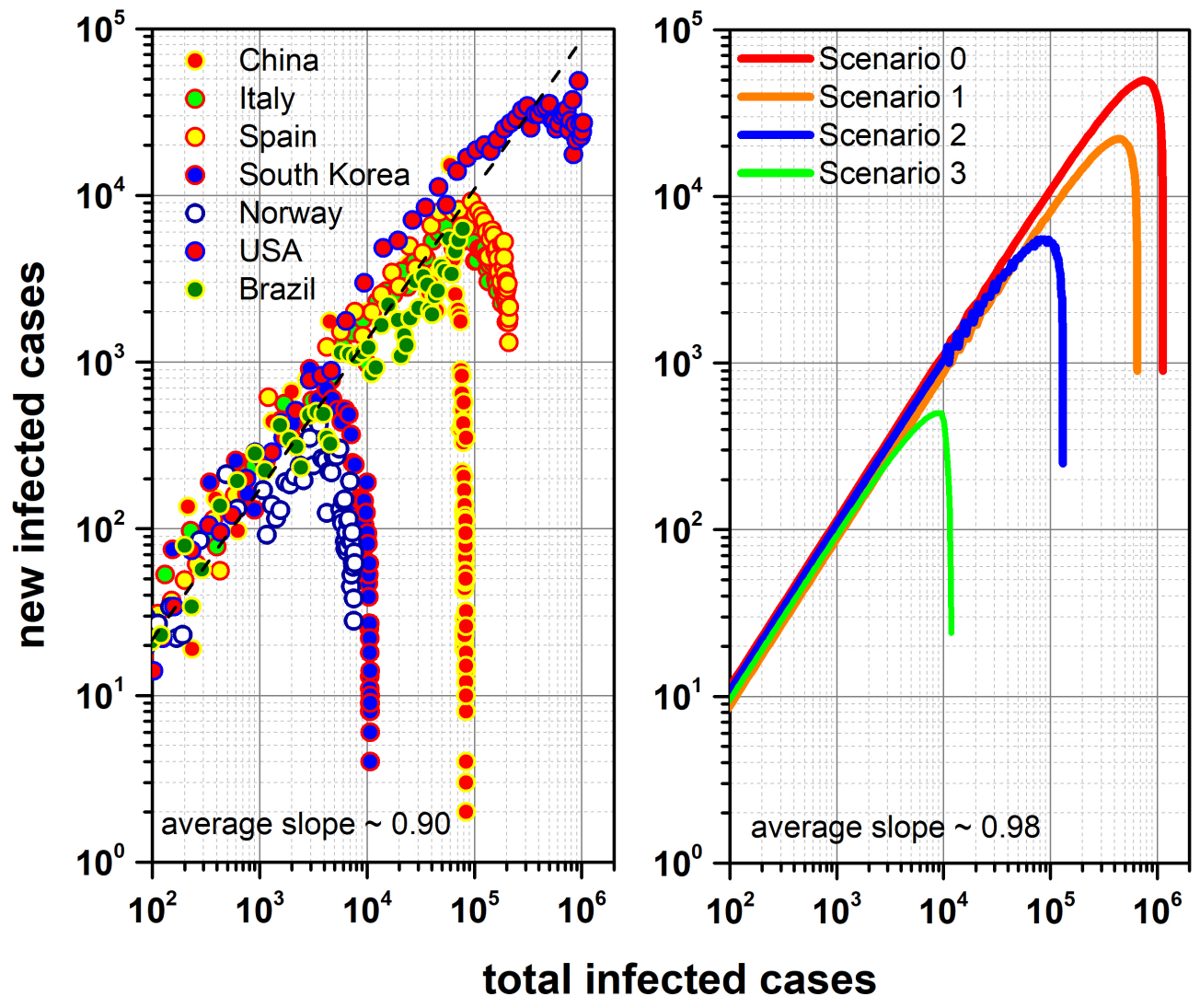

Figure 4. Curves representing the potential growth of new cases versus cases confirmed daily. Left: real data for all studied countries, right: model for the "simulated Brazilian scenarios". For the model, the colours from red to green represent scenarios $0,1,2$ and 3, respectively. Each country ${ }^{20}$ is represented with different coloured circles. The control parameters used in simulations was: $\beta=0.8, \phi=10^{-4}$, and for the isolation rate was used $\sigma=0.1$.

$$
R_{0}=\frac{\beta(1+\kappa)+\omega}{2 \gamma+i \sigma}
$$

\section{Discussion}

The model reproduces with good approximation, the dynamics of the COVID-19 pandemic in several countries studied. It was possible to adjust the dynamics of the different countries studied among the four scenarios simulated. The studies that was implemented based on the results obtained from the simulations shows the importance of containment measures to slow down the spread of the epidemic. These measures guarantee the flattening of the infection peak and the delay in the moment of maximum occurrence, allowing a better and greater preparation of the health system. The evolution of the epidemic is different in each country. The measures adopted to contain the increase in the number of infections, as well as the time until their application, are determining factors. The classification of countries according to the scenarios contemplated in the model depends on the criteria used for it. The basic reproduction rate for all simulated scenarios is calculated from the model. This parameter depends, in each scenario, on the parameters used in the model, as well as the measures that are implemented to delay the progress of the epidemic. The simulations show that more promising scenarios represent lower $R_{0}$ values, leading to less expressive and more delayed spikes of infection, all in correspondence with what the real data disclosed by the competent health entities show. 


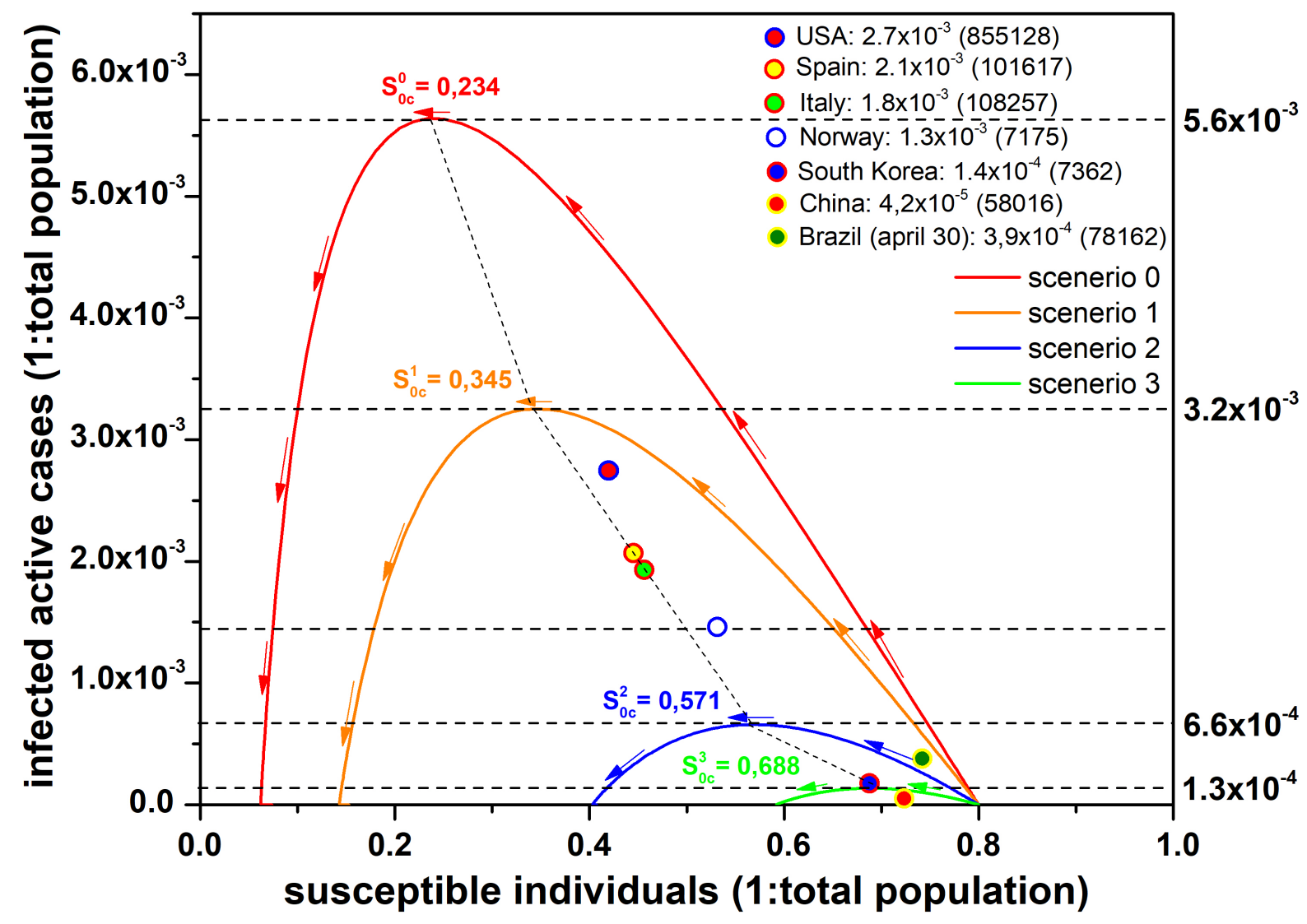

Figure 5. Phase diagram. The coloured circles represents the position of the maximums of the curve corresponding to each country, based on proportion of real active cases in relation to total population.

\section{Methods}

\section{Adapted model based on Ordinary Differential Equations (ODE).}

For countries where the virus was "imported", the original model is modified to exclude the host and the vector, taking into account the dynamics from several people initially infected with transmission capacity. To better illustrate the interactions between model variables, we can use the scheme of Figure 6. This scheme shows the interactions between all variables from deterministic rules. The system of differential equations that describe these interactions, without including isolation and quarantine actions, is as follows (scenario 0):

$$
\begin{aligned}
& \frac{d S(t)}{d t}=\alpha-\phi S(t)-\beta S(t)\left[I_{s}(t)+\kappa I_{a}(t)\right] \\
& \frac{d E(t)}{d t}=\beta S(t)\left[I_{s}(t)+\kappa I_{a}(t)\right]-(1-\delta) \omega E(t)-\delta \omega E(t)-\phi E(t) \\
& \frac{d I_{s}(t)}{d t}=(1-\delta) \omega E(t)-\gamma I_{s}(t)-\phi I_{s}(t)
\end{aligned}
$$




\begin{tabular}{|l|l|l|}
\hline Scenario & $\sigma$ & $R_{0}$ \\
\hline 0 & 0 & 4.04 \\
\hline 1 & 0.1 & 3.13 \\
\hline 1 & 0.2 & 2.25 \\
\hline 1 & 0.3 & 2.16 \\
\hline 2 & 0.1 & 2.55 \\
\hline 2 & 0.2 & 1.87 \\
\hline 2 & 0.3 & 1.47 \\
\hline 3 & 0.1 & 2.16 \\
\hline 3 & 0.2 & 1.47 \\
\hline 3 & 0.3 & 1.12 \\
\hline
\end{tabular}

Table 3. Basic reproduction number values in each scenario and for each value of $\sigma$. The transmission rate $\beta=0.8$ was used.

$$
\begin{aligned}
& \frac{d I_{a}(t)}{d t}=\delta \omega E(t)-\gamma I_{a}(t)-\phi I_{a}(t) \\
& \frac{d R(t)}{d t}=\gamma I_{s}(t)+\gamma I_{a}(t)-\phi R(t)
\end{aligned}
$$

In the model represented here, there are three groups of individuals: susceptible and exposed ( $\mathrm{S}$ and $\mathrm{E}$ ), infected symptomatic and asymptomatic $\left(I_{s}\right.$ and $\left.I_{a}\right)$ and recovered and/or killed (R). Susceptible individuals are exposed due to their interaction with infected individuals of both types, with different degree of exposure. Infection of symptomatic patients occur from exposed individuals, after the virus incubation period, with a certain probability; asymptomatic patients, on the other hand, happen from individuals who have been exposed and who have latent viral particles in their body. A percentage of all individuals who have been infected will be recovered and the removal of individuals of each type happens naturally and is related, in addition to death, to the "exit" of the system. The virus incubation period reported in ${ }^{15}$ is 5.2 days. As in the original model, we assume that this is equally your latency period, so $\omega=0.1923$. We also use the infection period value reported by the original study, so $\gamma=$ 0.1724 . The values of the proportion of asymptomatic infection, $\delta=0.5$ and the multiple of the transmissibility of $I_{a}$ to $I_{s},=0.5$ are the same as in the original model. The removal rate $\phi$ depends on each country. In our model, it was considered a control parameter as well as the transmission rate $\beta$, which depends directly on actions by the population and the state, for adjusting the curves to the dynamics of all countries. The system of Equations $(2-6)$. indicates the dynamics for the most pessimistic scenario, where containment measures against the advance of the epidemic such as quarantine or population isolation are not adopted (scenario 0). The inclusion in our model of actions to contain the epidemic can be seen in the scheme of Figure 6 . In this scheme, these measures are represented with the following colours: blue, quarantine of symptomatic infected, red, isolation of exposed individuals and green, the isolation of asymptomatic infected people. The system considering only the quarantine of the symptomatic infected represents scenario 1, the system including scenario 1 plus the isolation of exposed individuals represents scenario 2 and the inclusion, in addition to the other two scenarios, of the isolation of asymptomatic individuals, represents scenario 3, that would be the most optimistic scenario, but less realistic. In scenarios 1 to 3 , the isolation or quarantine of symptomatic infected individuals, exposed persons, and asymptomatic infected individuals, respectively, is proposed. Each scenario accumulates the measurements of the previous one with its own. In the model, these actions are represented by adding the terms $-\sigma I_{s}(t),-\sigma E(t)$ and $-\sigma I_{a}(t)$ in equations 3,4 and 5 , respectively, as well as, the same terms, but positive, in equation 6 , for each specific scenario. When simulating scenarios 1 to 3 , the specific terms of removal of each variable, $\phi I_{S}(t), \phi E(t)$ and $\phi I_{s}$, do not appear in the system of equations. The isolation condition considers that the variable in question cannot "leave" the system. The values of the parameters used in the simulations are shown in table 4.

\section{References}

1. Oliveira, E. \& Ortiz, B. Ministério da saúde confirma primeiro caso de coronavírus no brasil. figshare https://g1.globo. $\mathrm{com} /$ ciencia-e-saude/noticia/2020/02/26/ministerio-da-saude-fala-sobre-caso-possivel-paciente-com-coronavirus.ghtml (2020).

2. A.K., S., E., S. \& van Hils A.S. A two-age-classes denge transmission model. Math Biosci 216, 114 - 121 (2008). 


\begin{tabular}{|l|l|l|l|}
\hline Parameter/Initial condition & Description & Value & Scenario \\
\hline$\alpha$ & Replacement of susceptible individuals rate & $8.10^{-5}$ & $0-3$ \\
\hline$\phi$ & Removal/replacement rate (control parameter) & Variable & $0-3$ \\
\hline$\beta$ & Transmission rate (control parameter) & Variable & $0-3$ \\
\hline$\kappa$ & Multiple of the transmissibility of $I_{a}$ to $I_{s}$ & 0.5 & $0-3$ \\
\hline$\delta$ & Proportion of asymptomatic infection rate & 0.5 & $0-3$ \\
\hline$\omega$ & Incubation frequency & $0.1923^{15}$ & $0-3$ \\
\hline$\gamma$ & Latency frequency & $0.1724^{15}$ & $0-3$ \\
\hline$\sigma$ & Proportion of individuals in quarantine/isolation & Variable $^{-}$ & $1-3$ \\
\hline $\mathrm{S}(0)$ & Initial proportion of susceptible individuals & 0.8 & $0-3$ \\
\hline $\mathrm{E}(0)$ & Replacement of exposed individuals & 0.0 & $0-3$ \\
\hline$I_{S}(0)$ & Replacement of symptomatic individuals & $5.10^{-7}$ & $0-3$ \\
\hline$I_{a}(0)$ & Replacement of asymptomatic individuals & 0.0 & $0-3$ \\
\hline $\mathrm{R}(0)$ & Initial proportion of removed individuals & 0.0 & $0-3$ \\
\hline
\end{tabular}

Table 4. List of parameters and initial conditions used in the simulations. The replacement of susceptible individuals rate $\alpha$ is considered as $\phi \mathrm{S}(0)^{15}$. For the proportion of individuals in quarantine/isolation $\sigma$ were used values of $0.1,0.2$ and 0.3 , simulating different degrees of adhesion.

3. S.N., G., A.B., G. \& M.R., A. B. Backward bifurcations in dengue transmission dynamics. Math Biosci 215, $11-25$ (2008).

4. M., D. \& A., B. Dengue fever: Mathematical modelling and compjuter simulation. Appl Math Comput. 117, 528 - 544 (2006).

5. L.C.d.C., M. et al. Modelling the dynamics transmission of dengue fever: Investigating disease persistence. PLoS Negl Trop Dis. 5, 1 (2011).

6. A.S., P. \& R.M., R. Hepatitis b virus kinetics and mathematical modeling. Sem. Liv. Dis. 24, 11 - 15 (2004).

7. A.S., P. Modelling viral and immune system dynamics. Nat. Rev. Immunol. 2, 28 - 36 (2002).

8. M., N. et al. Viral dynamics in hepatitis b infection. Proc. Natl Acad. Sci.USA. 93, 4398 - 4402 (1996).

9. Gharib-Zahedi, M. R. \& Ghaemi., M. Kinetics of hepatitis b virus infection: A cellular automaton model study. J. Paramed. Sci. (JPS). 3, 2008 - 4978 (2012).

10. Rogers., D. The dynamics of vector-transmitted diseases in human comunities. Philos Trans $R$ Soc Lond B Biol Sci. 321, $513-539$ (1998).

11. E., A. \& H.N., A. On modelling epidemics. including latency, incubation and variable susceptibility. Phisica A. 253, 347 352 (1998).

12. E., A., H.N., A. \& S.Z., H. On modelling hepatitis b transmission using cellular automata. J. Stat. Phys. $92,707-712$ (1998).

13. Gomez, S., Arenas, A., Borge-Holthoefer, J., Meloni, S. \& Moreno., Y. Discrete-time markov chain approach to contact-based disease spreading in complex networks. EPL (Europhysics Lett. 89.

14. Gomez, S., Gómez-Gardenes, J., Moreno, Y. \& Arenas., A. Nonperturbative heterogeneous mean-field approach to epidemic spreading in complex networks. Phys. Rev. E. 84.

15. Chen, T.-M. et al. A mathematical model for simulating the phase-based transmissibility of a novel coronavirus. Infect. Deseases Poverty 9, 24, DOI: https://doi.org/10.1186/s40249-020-00640-3 (2020).

16. Arenas, A. et al. A mathematical model for the spatiotemporal epidemic spreading of covid19 (preprint). medRxiv DOI: https://doi.org/10.1101/2020.03.21.20040022 (2020).

17. Squillante, L., Mello, I. F., Seridonio, A. C. \& de Souza., M. Attacking the covid-19 with the ising-model and the fermi-dirac distribution function (preprint). arRxiv:2003.11860v1 [q-bio.PE] (2020).

18. Crokidakis., N. Data analysing and modelling of the evolution of covid-19 in brazil (preprint). arRxiv:2003.12150v1 [q-bio.PE] (2020).

19. Shulz, R. A., Coimbra-Araújo, C. H. \& Costiche., S. W. S. Covid-19: A model for studyng the evolution of contamination in brazil (preprint). arRxiv:2003.13932v1 [q-bio.PE] (2020). 


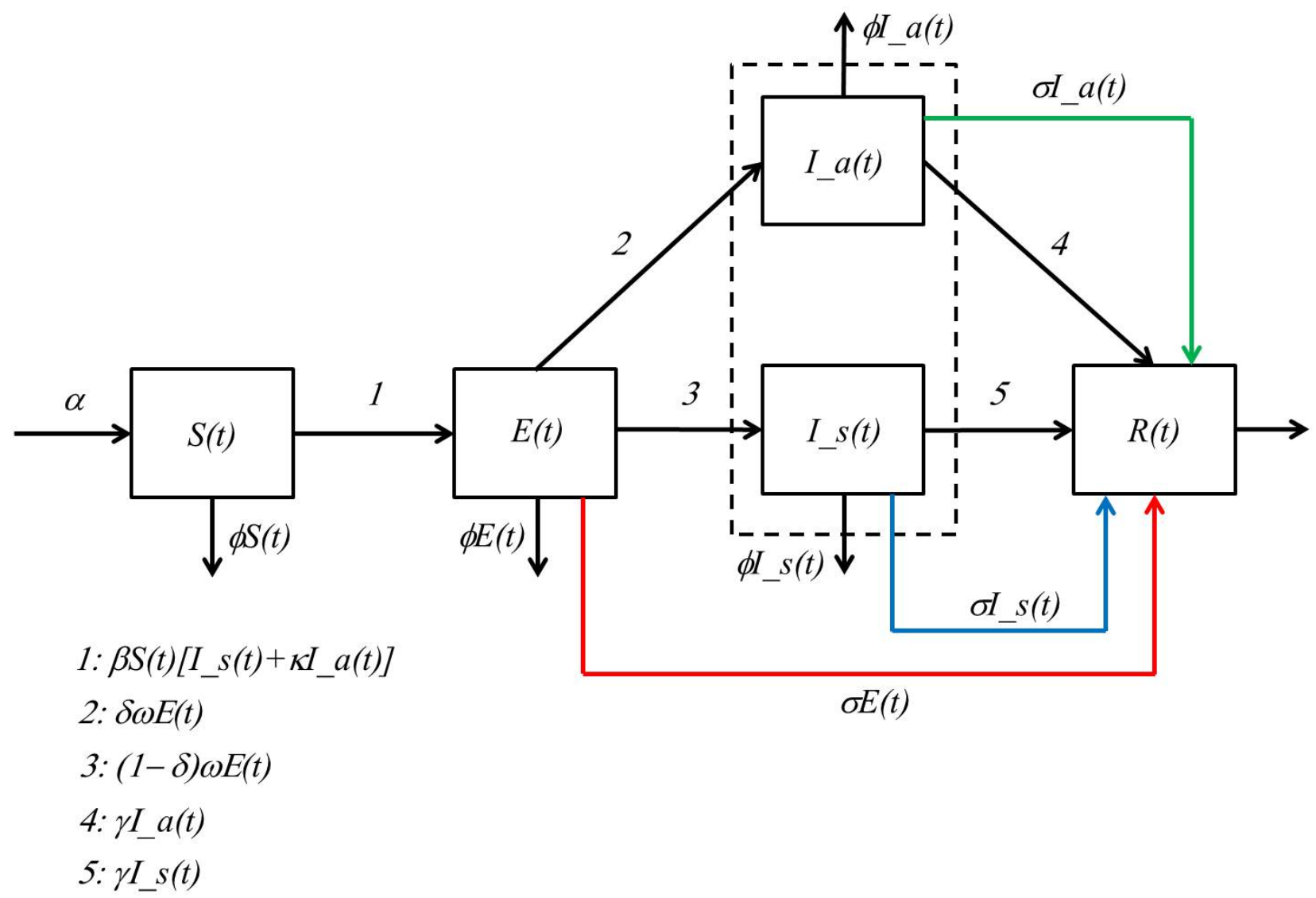

Figure 6. Representative scheme of the interactions between the different variables of the model.

20. Ritchie, H. Coronavirus source data. figshare https://ourworldindata.org/coronavirus-source-data (2020).

21. (MinutePhysics), H. How to tell if we're beating covid-19. figshare https://www.youtube.com/ (2020).

\section{Acknowledgements}

I thank Fernando de León Pérez for the critical Reading of the manuscript.

\section{Author contributions}

All authors contributed equally in the discussions regarding this work. R.E.R.G. modeled the system, performed the simulations, made the graphics, and wrote this manuscript. M.A.Z.M, C.E.L and C.A.C.M. read and revised the manuscript.

\section{Additional information}

Correspondence should be addressed to R.E.R.G.

\section{Competing interests}

The authors declare no competing interest. 


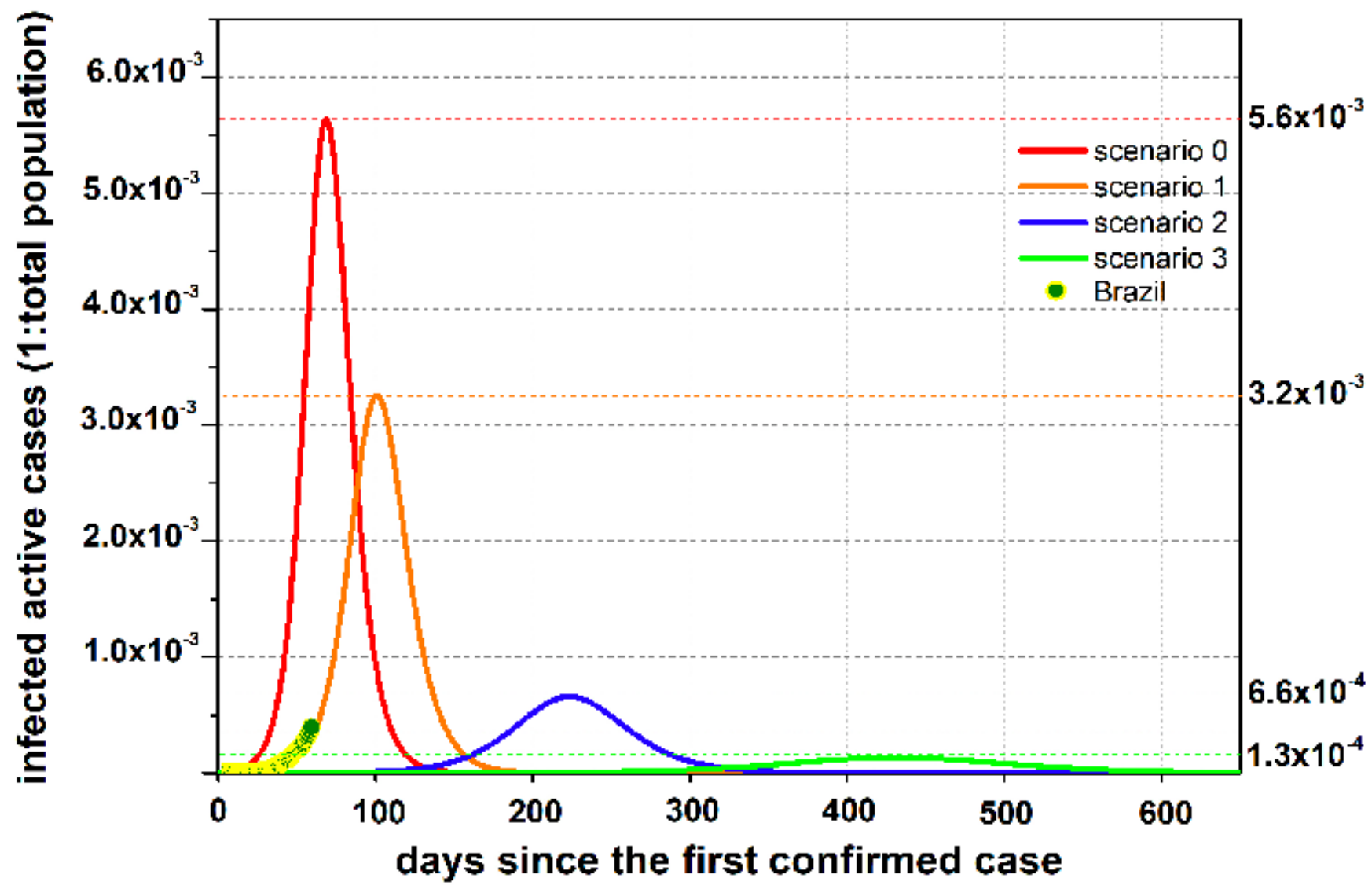

Figure 1

Total infected cases versus time in days, for all scenarios. In red, the dynamic corresponding to the worst scenario is represented, while the most optimistic scenario is represented in green. Vertical axis represent the reported values of infected cases. Real data for Brazil until April 30 are represented with coloured circles. The control parameters used in simulations was: $\beta=0.8, \Phi=10^{\wedge}-4$, and for the isolation rate was used $\sigma=0.1$. 


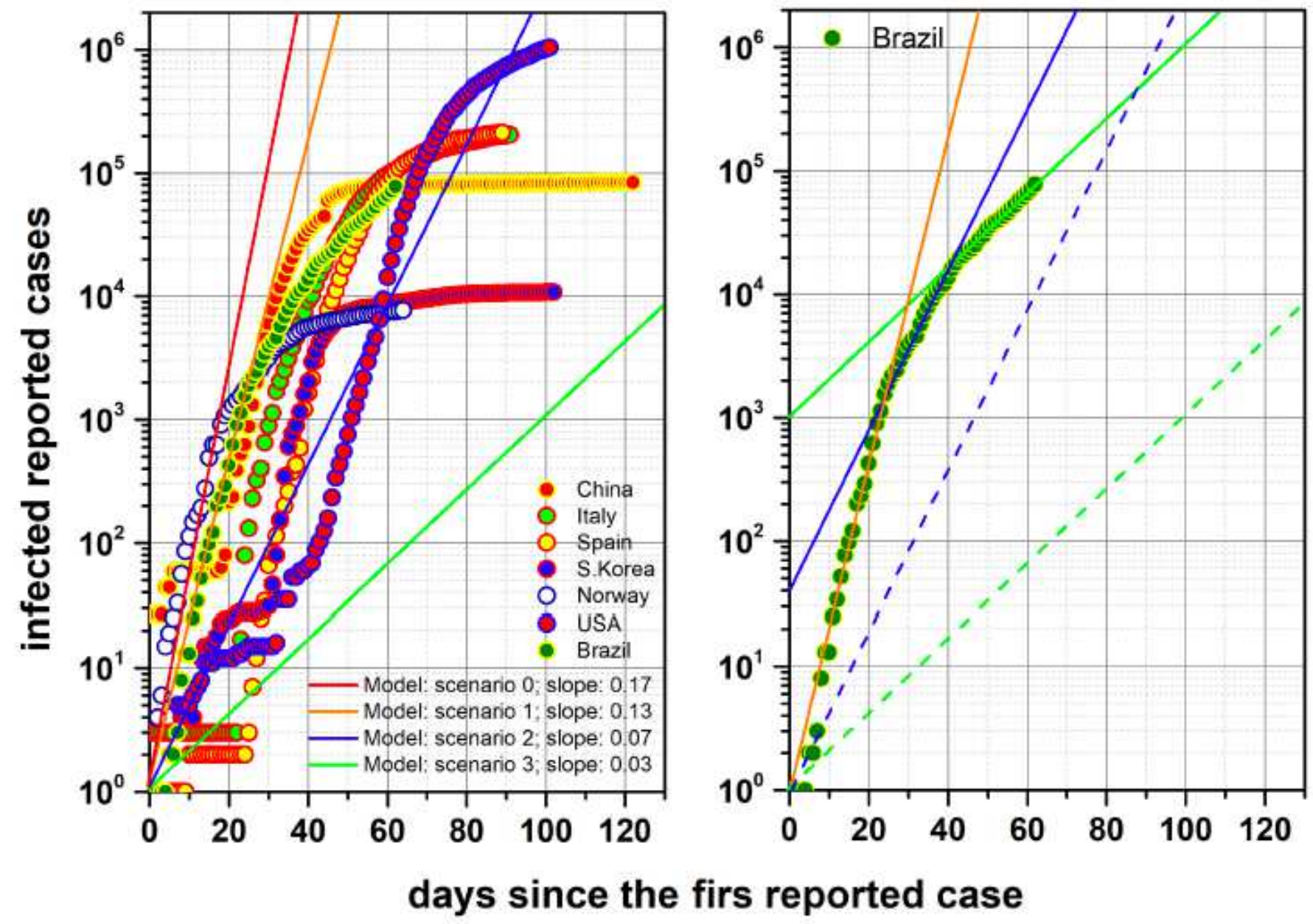

Figure 2

Representative curves of the exponential growth. The colours from red to green represent scenarios $0,1,2$ and 3 respectively. Real confirmed cases for each country20 are represented with different coloured circles, in the left panel, and for Brazil in the right panel. The control parameters used in simulations was: $\beta=0.8, \Phi=10^{\wedge}-4$, and for the isolation rate was used $\sigma=0.1$. 

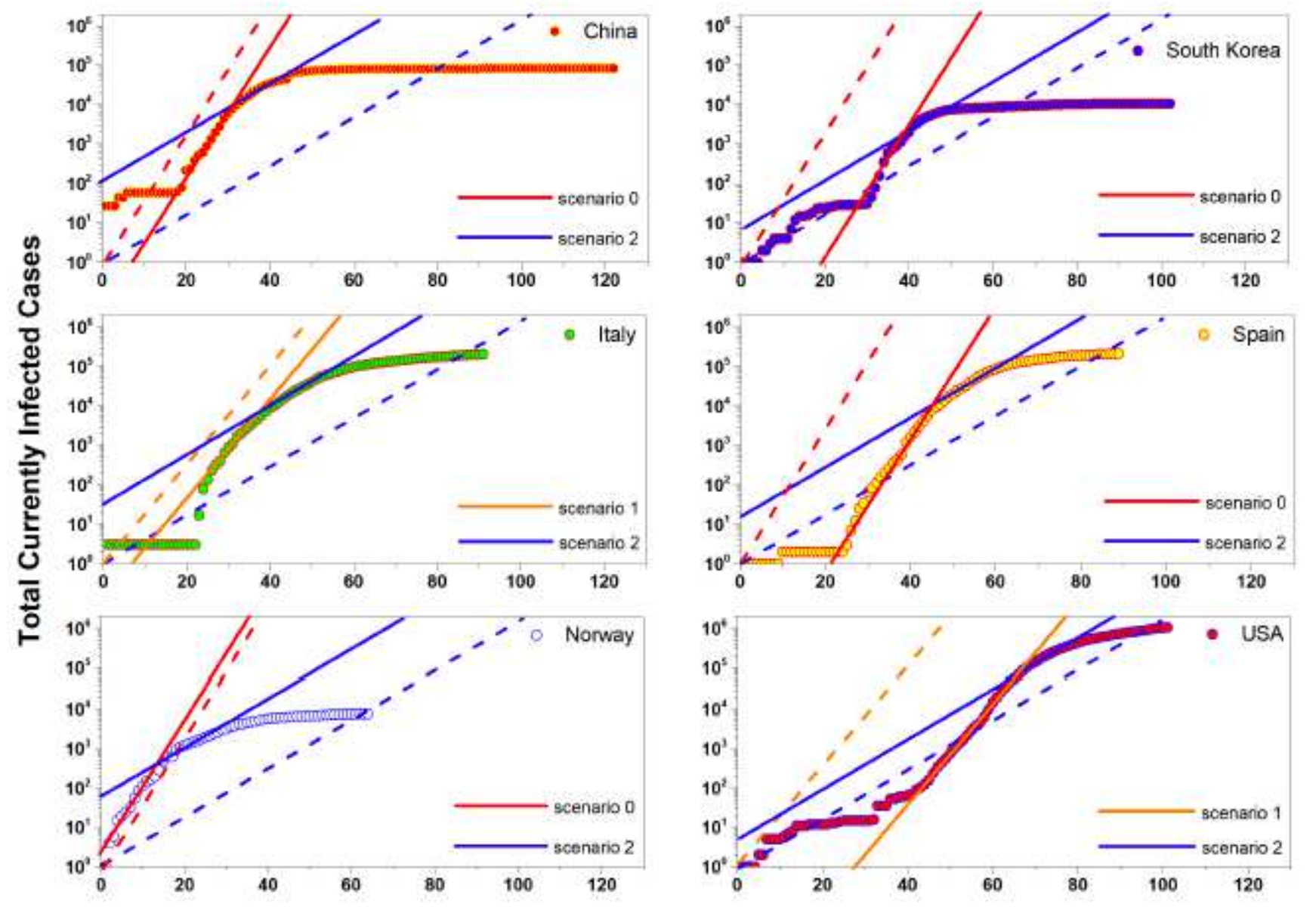

Days since the first reported case

\section{Figure 3}

Representative curves of the exponential growth. Different countries are represented in each panel. The scenarios corresponding to each stage of progression of the epidemic are represented with colored lines. The control parameters used in simulations was: $\beta=0.8, \Phi=10^{\wedge}-4$, and for the isolation rate was used $\sigma$ $=0.1$. 


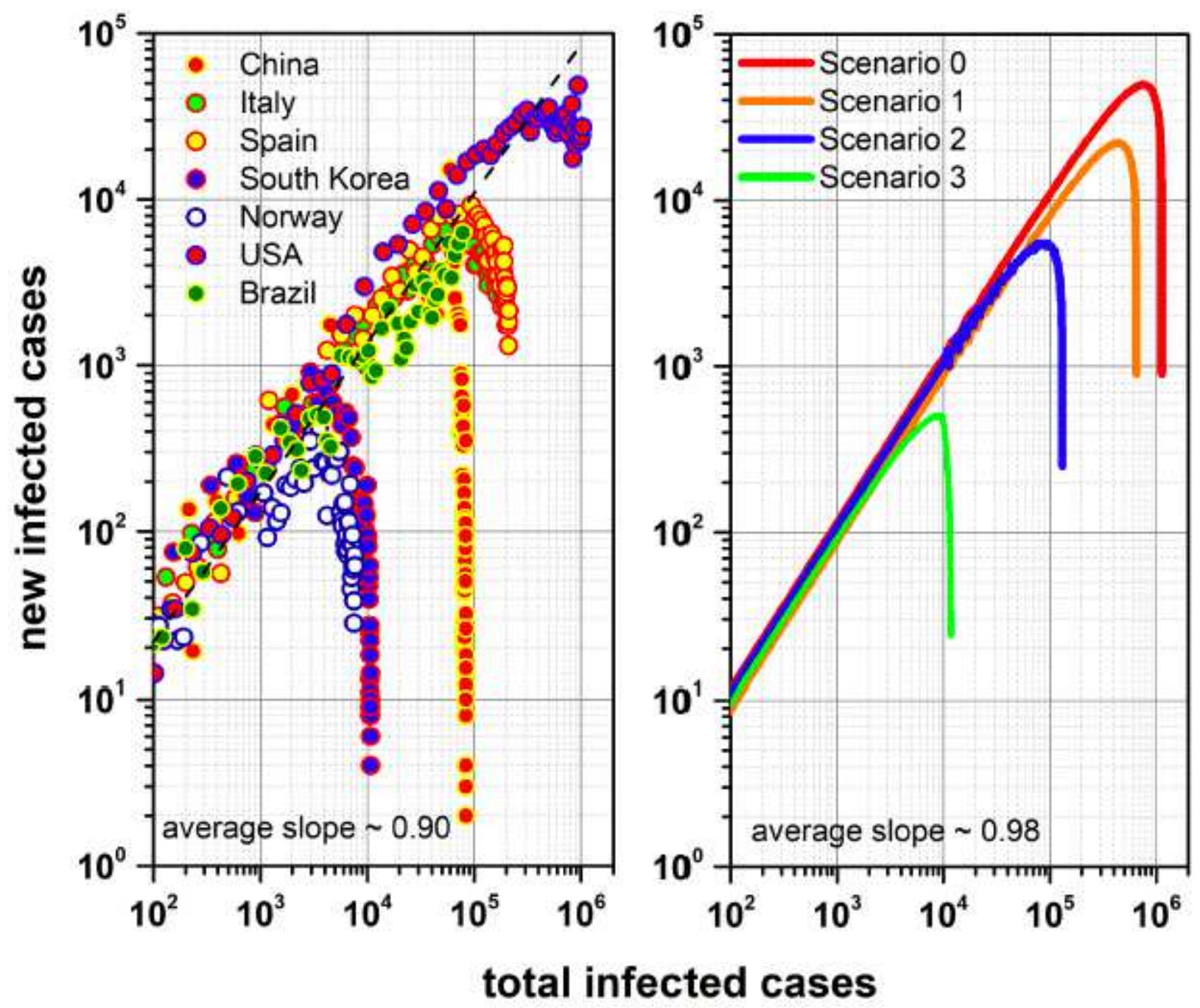

Figure 4

Curves representing the potential growth of new cases versus cases confirmed daily. Left: real data for all studied countries, right: model for the "simulated Brazilian scenarios". For the model, the colours from red to green represent scenarios $0,1,2$ and 3 , respectively. Each country20 is represented with different coloured circles. The control parameters used in simulations was: $\beta=0.8, \Phi=10^{\wedge}-4$, and for the isolation rate was used $\sigma=0.1$. 


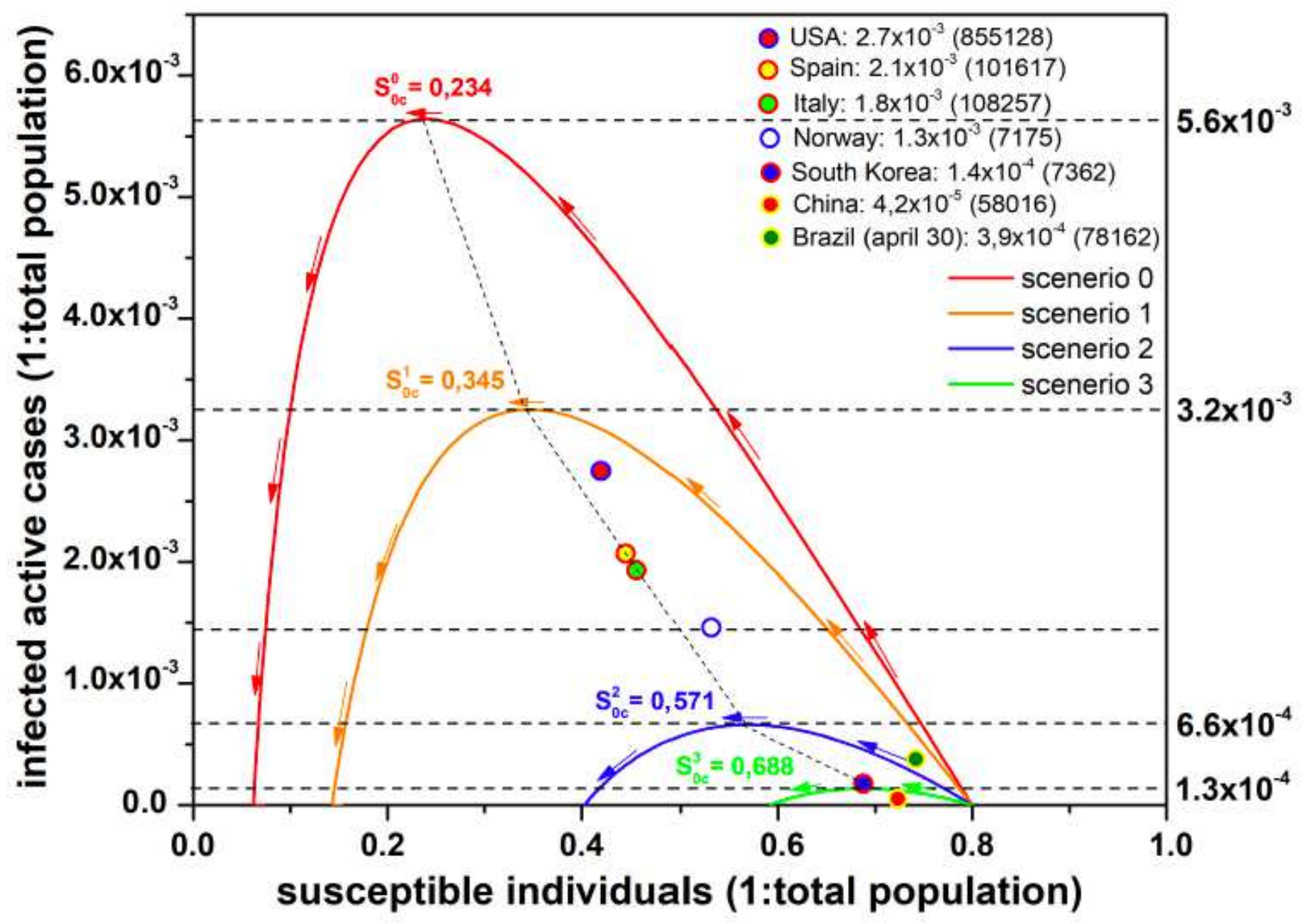

Figure 5

Phase diagram. The coloured circles represents the position of the maximums of the curve corresponding to each country, based on proportion of real active cases in relation to total population. 


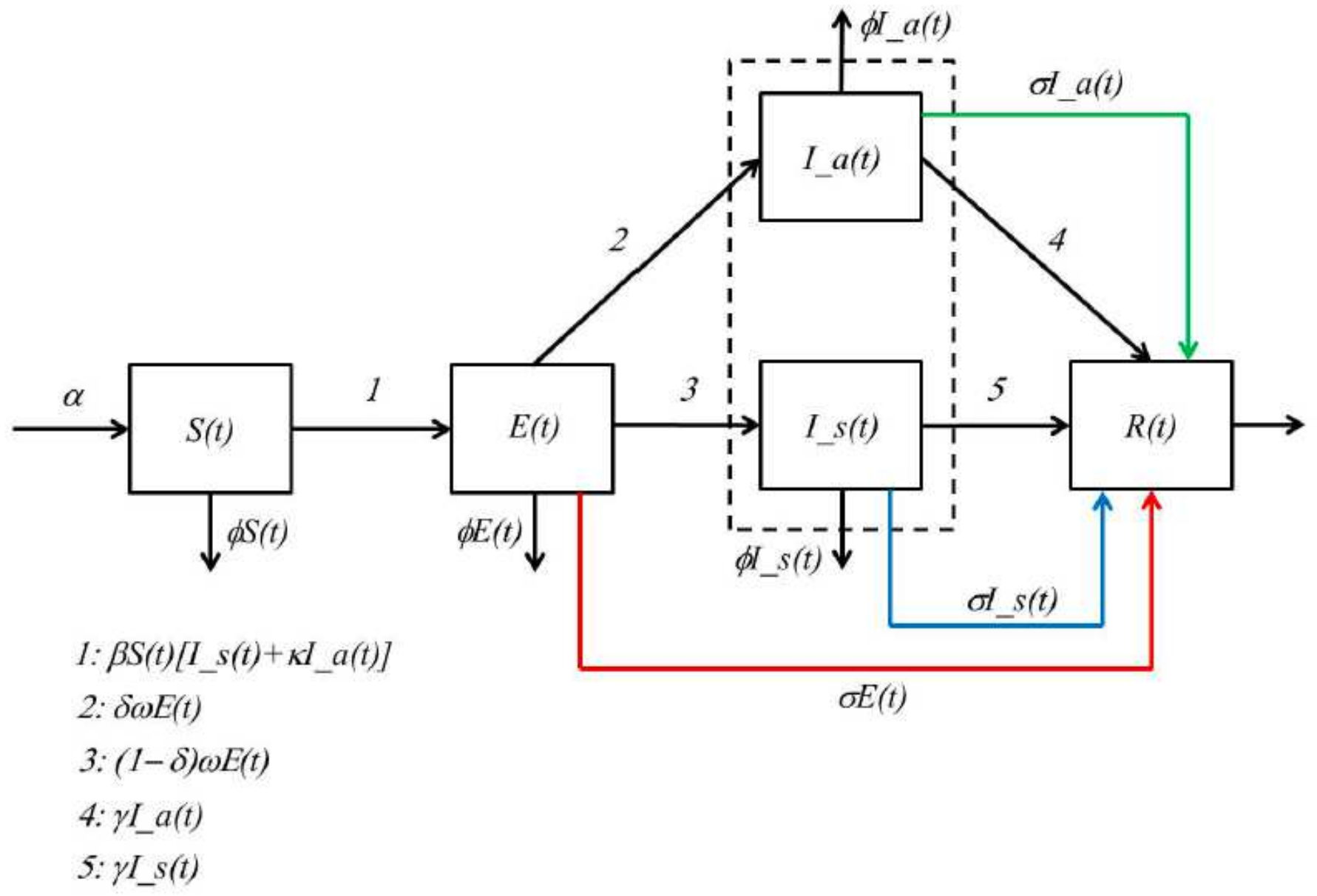

Figure 6

Representative scheme of the interactions between the different variables of the model. 